\title{
Jet quenching in high energy nuclear collisions and quark-gluon plasma.
}

\author{
Yu.A. Tarason* \\ Russian Research Center "Kurchatov Institute", 123182, Moscow, Russia
}

(Dated: 24th November 2018)

\begin{abstract}
We investigate the energy loss of quark and gluon jets in quark-gluon plasma produced in the central $\mathrm{Au}+\mathrm{Au}$ collisions at RHIC energy. We use the physical characteristics of initial and mixed phases found in the effective quasiparticle model [5]. We also take into account the possibility of hot glue production at the first stage. We calculate the suppression of $\pi^{0}$ spectrum, which is caused by the energy loss of gluon and quark jets. We compare this suppression with data reported by PHENIX. We find that the suppression is described correctly by the quasiparticle model with decrease of thermal gluon mass and effective coupling in the region of phase transition (at $T \rightarrow T_{c}$ from above). Thus it is possible to investigate the structure of phase transition with the help of hard processes. We also show, that the energy loss at SPS energy is too small to be observable. This is caused, in fact, by too low initial temperature of the plasma phase at this energy.
\end{abstract}

PACS numbers: $12.38 . \mathrm{Mh}, 24.85 .+\mathrm{p}, 25.75 .-\mathrm{q}$

*Electronic address: tarasov@dni.polyn.kiae.su 


\section{INTRODUCTION}

Energy loss of high energy quark and gluon jets in relativistic $\mathrm{A}+\mathrm{A}$ collisions leads to jet quenching and thus probes the quark-gluon plasma [1]. Recently the considerable suppression of hadron spectra at moderately high $p_{\perp}<6 \mathrm{GeV}$ was found in the central $\mathrm{Au}$ + Au collisions, especially for $\pi^{0}$ production [2]. This suppression can be explained by the energy loss of the moderate $p_{\perp} \leq 10 \mathrm{GeV}$ gluons jets. This is one of the major new results at RHIC energy, which has not been observed before at lower SPS energy. The energy loss was investigated in the work [3] in various orders in opacity $L / \lambda_{g}$ (where $L$ is nuclear radius and $\lambda_{g}$ is the gluons mean free path). It was shown that these series is strongly dominated by the first term. It was also shown in Ref. 4] that finite kinematic boundaries decrease the energy loss at intermediate jet energies as compared to the asymptotic limit. Recently, in paper [5], the physical characteristics of initial plasma phase and also of mixed phase at SPS and RHIC energies (i.e. the values $T_{0}, \tau_{0}, \tau_{c}$ ) were investigated on the basis of the quasiparticle model and isentropic expansion. In the present work we use these initial conditions for investigation of the energy loss of high energy gluon and quark jets at RHIC energy. In these studies we take into account also the possibility of two stages of equilibrium, i.e. production of the hot glue at first stage [6]. The calculation of energy loss is important for investigation

of the phase transition structure of quark-gluon plasma into hadrons. In the Refs. [7, 8] the phenomenological parametrization of coupling constant $G(T)$ was used in accordance with new lattice data. When approaching to phase transition point from above, the decrease of thermodynamic values is caused, according to $S U(3)$ gauge theory, by increase of the thermal gluon mass and also of the coupling constant $G(T)$. In these conditions the applicability of perturbation theory is questionable. In phenomenological model of confinement [9] the decrease of thermal gluon mass $m_{g}(T)$ at $T \rightarrow T_{c}$ from above is assumed, which is connected with decrease of effective coupling strength $G(T)$. It can be understood, as the more and more gluons become confined and form heavy glueballs with decrease of $\mathrm{T}$, and the effective glueball exchange interaction between gluons are reduced. However, in this case the entropy density $s(T)$ will exceed the lattice entropy because of domination of light masses near $T_{c}$. This difference may be accounted for in quasiparticle model by modification of the number of effective degrees of freedom in thermodynamic functions:

$$
g_{g} \rightarrow C(T) g_{g}
$$


The explicit value $C(T)$ may be estimated as ratio of the lattice entropy and the quasiparticle entropy density $s_{g}(T)$ with decrease of the mass $m_{g}(T)$. At $T \gg T_{c}$ we have $C(T) \simeq 1$, and near $T \sim T_{c}$ we have $C(T)<1$. The value $C(T)$ for gluons has the form $[9]$ :

$$
C\left(T, T_{c}\right)=C_{0}\left(1+\delta_{c}-\frac{T_{c}}{T}\right)^{\beta_{c}},
$$

where $C_{0} \simeq 1.25, \delta_{c} \simeq 0.0026, \beta_{c} \simeq 0.31$. The thermal mass $m_{g}(T)$ can be parameterized well by formula:

$$
m_{g}(T)=\left(\frac{N_{c}}{6}\right)^{1 / 2} G_{0} T\left(1+\delta-\frac{T_{c}}{T}\right)^{\beta},
$$

where $\beta \simeq 0.1, \delta \simeq 10^{-6}$. It possible to vary some parameters, for example $\beta \simeq 0.05, \delta \simeq$ $10^{-7}$ are reasonable 9 ]. The form (3) is analogous to behaviour of Debye mass $m_{D}$, extracted from lattice data $[\underline{8}, 9]$. The value $G_{0}$ can be determined by asymptotic value of the thermal mass, chosen from coincidence of lattice and perturbative masses at $T \simeq 3 T_{c}[$, , 8]. We had found for pure gluons: $G_{0} \simeq 1.9[\underline{5}$.

The relations (21), (3) with above-mentioned parameters give good description of $S U(3)$ lattice data for entropy $s$, energy density $\epsilon$, and pressure $p$ for $T$ close to $T_{c}$. It is possible to extend the effective quasiparticle model to a system with dynamic quarks, with analogous effective coupling $G(T)$, and the function $C\left(T, T_{c}\right)$ with some variation of parameters [9]. One can show that the same constant $G_{0} \simeq 1.9$ describes the lattice data for 2 and $2+1$ flavors (for example, the lattice pressure [5, 8].

It is interesting to note, that the massive constituent quarks $\left(m_{q}\right.$ and $\left.m_{s}\right)$ appears also due to the decrease of number of degrees of freedom in the presence of octet of pseudogoldstone states. This is a consequence of conservation of the entropy and of the number of net nucleons. It can also be demonstrated that hadrons (in the hadronic part of the mixed phase) appears with the same effective number of degrees of freedom [5].

It can be shown, that such picture of phase transition (with decrease of effective coupling strength $G(T)$ near $T \simeq T_{c}$ ) gives reasonable quantitative description of the jet quenching and of suppression of the hadronic spectra in the central $\mathrm{Au}+\mathrm{Au}$ collisions at RHIC energy. However, the use of the "running" coupling $\alpha_{s}(T)$ in the perturbative decomposition of the thermodynamic values in plasma leads to too large energy loss of jets, which disagrees with experimental data for suppression of the hadrons with large $p_{\perp}$ at RHIC energy.

In the Ref. [5] we do not find a noticeable difference for meson and baryon spectra in the ordinary perturbative theory with the running coupling $\alpha_{s}(T)$ and also in model with 
phenomenlogical parametrization of coupling $G(T)$ [7, 8] in comparison with the effective quasiparticle model. However, the ordinary perturbative model disagrees with $S U(3)$ lattice data in the region of phase transition. Thus the spectra of particles apparently weakly depend on the character of phase transition. Therefore the investigation of jet quenching represent significant interest.

In Sec. II] we calculate the energy loss of the high energy gluon and quark jets in quarkgluon plasma at RHIC energy. In these calculations we use the initial conditions in plasma at SPS and RHIC energies, which were found in the quasiparticle model [5]. We also take into account here the production of hot glue at the first stage. We show that the energy loss at SPS energy is too small. We show also, that in the perturbative theory the energy loss of gluon jets is too large.

In Sec. III we calculate the suppression of $\pi^{0}$ at moderate $p_{\perp}\left(3 \leq p_{\perp} \leq 6\right) \mathrm{GeV}$ in the central $\mathrm{Au}+\mathrm{Au}$ collisions. We take into account the jet quenching and the parton shadowing factor in nucleus. We show that suppression of the $\pi^{0}$ spectra at $P_{\perp}^{\pi^{0}} \geq 3 \mathrm{GeV} / \mathrm{c}$ does not contradict the experimental data at RHIC energy.

\section{ENERGY LOSS OF HIGH ENERGY JETS IN QUARK-GLUON PLASMA}

In the effective quasiparticle model [5] we have investigated the initial condition and the evolution of the plasma stage, where there is equilibrium for both quarks and gluons. We have found for RHIC case the values $T_{0} \simeq 216.3-219.6 \mathrm{MeV}, \tau_{0} \simeq 2.22-2.18 \mathrm{fm}$ and $\tau_{c} \simeq 6.34-6.52 \mathrm{fm}$. We have also found corresponding values for SPS energy $T_{0} \simeq 175$ $\mathrm{MeV}, \tau_{0} \simeq 3.28 \mathrm{fm}, \tau_{c} \simeq 4.1 \mathrm{fm}$, so, here we have the short plasma stage. The production of a more hot glue plasma at the first stage is caused by the relatively large $g g$ cross section in comparison with the $q g$ and $q q$ cross section of Ref. 6]. In the lowest order, the matrix elements $M^{2}$ in formula:

at large angles are related thus as [10]:

$$
\frac{d \sigma}{d t}=\frac{\pi \alpha_{s}^{2}}{s^{2}} M^{2}
$$

$$
M_{g g \rightarrow g g}^{2} / M_{q g \rightarrow q g}^{2} / M_{q q \rightarrow q q}^{2}=30.4 / 5.4 / 2.2
$$

i.e. $g g$ scattering is most important. The small angle scattering leads to divergent crosssections, which however are finite in $Q G P$ due to the finite "Debye mass" $t_{\min }=g^{2} T^{2}$. The 
cross section at large angles is defined by formula:

$$
\sigma_{g g}^{\text {large-angle }} \simeq \frac{30.4 \pi \alpha_{s}^{2}}{2 \bar{s}} \simeq \frac{95.46 \alpha_{s}^{2}}{36 T^{2}} \simeq 2.65 \frac{\alpha_{s}^{2}}{T^{2}}
$$

The cross section at small angles has the form:

$$
\sigma_{g g}^{\text {small-angle }} \simeq \frac{9}{2} \frac{\pi \alpha_{s}^{2}}{4 \pi T^{2} \alpha_{s}}=\frac{9}{8} \frac{\alpha_{s}}{T^{2}}
$$

The effective scattering rate $1 / \tau_{g}$ is determined by a sum of large and small angle values:

$$
\frac{1}{\tau_{g}}=n_{g}\left(2.65 \frac{\alpha_{s}^{2}}{T^{2}}+\frac{9}{8} \frac{\alpha_{s}}{T^{2}}\right) .
$$

Here the value $\alpha_{s}$ is determined by the effective coupling constant $\alpha_{s}=\frac{G^{2}}{4 \pi}$, where:

$$
G\left(T, T_{c}\right)=G_{0}\left(1+\delta-\frac{T_{c}}{T}\right)^{\beta}
$$

and $G_{0} \simeq 1.9$ (see Eq. (3in)

The net gluon density in effective quasiparticle model is:

$$
n_{g}(T)=\frac{16 T^{3}}{2 \pi^{2}} \int_{0}^{\infty} d x \frac{x^{2} C\left(T, T_{c}\right)}{e^{\sqrt{x^{2}+\frac{m_{g}^{2}}{T^{2}}}-1}},
$$

where

$$
\frac{m_{g}^{2}}{T^{2}}=\frac{1}{2}\left[G_{0}\left(1+\delta-\frac{T_{c}}{T}\right)^{\beta}\right]^{2} .
$$

To estimate the initial temperature of the hot glue we use Bjorken model [11]. The value $d N / d y$ (number of charged and neutral particles per unit central rapidity) for $A u+A u$ collisions can be described by formula

$$
\left(\frac{d N}{d y}\right)_{y=0} \simeq 0.8 \ln \sqrt{s} A_{A u}^{1.11} .
$$

This value agrees with experimental value 1374 at $\sqrt{s}=130 \mathrm{GeV}$.

In this paper we make the following assumptions: at the first stage we have the hot glue in equilibrium and nonequilibrium quarks. The total entropy of gluons and such quarks (at temperature $T_{g}$ ) is less than the entropy of gluons and all quarks at more a later stage (at above mentioned temperature $T_{0}$ ). With expansion of the system the temperature decreases and the entropy of quarks and gluons decreases (if to assume that all the quarks are nonequilibrium ones). However, in reality, some part of quarks achieves equilibrium and entropy 
increases. With subsequent decrease of $\mathrm{T}$ the number of equilibrium quarks increases, and near $T_{0}$ we have the equilibrium of both gluons and quarks. After that the cooling becomes isentropic.

One can estimate the fraction of gluons and sea $u, d, s$ quarks (and antiquarks) from the structure function [12], if to imagine the system moving backward in time from many secondaries into hot partonic plasma. This approach has to normalize parton multiplicity to the total entropy, later observed as multiplicity of secondaries. We assume its conservation at later stage (for $T \leq T_{0}$ ) and its additional production at previous stage [6]. For average number of partons (for example, gluons in nucleon) we use formula [13]:

$$
N_{g}=\int_{m_{\text {char }} /|p|}^{1} d x g(x, Q),
$$

where $g(x, Q)$ is structure function, $m_{\text {char }} \sim m_{\rho}$ is characteristic hadrons mass, and $|p|$ is nucleon momentum. For RHIC energies we have $m_{\text {char }} /|p|=2 m_{\text {char }} / \sqrt{s} \approx 0.012$.

Similarly, the number of the sea quarks is

$$
N_{\text {sea }}=2 \int_{m_{\text {char }} / p \mid}^{1} d x\left(u_{\text {sea }}+d_{\text {sea }}+s_{\text {sea }}\right) .
$$

This gives estimate $N_{\text {sea }} / N_{g} \approx 0.44$. This correspond to known fact, that gluons dominate in structure function at small $\mathrm{x}$ : the number of gluons is more than twice larger than the number of sea quarks and antiquarks. The characteristic average value $x_{\min } \simeq 0.012$ is close to value $x_{\min } \simeq 0.01$ in the works of other authors (for example E.V.Shuryak). These meanings are reasonable for estimation of the hot glue temperature $T_{g}$, since partons with $x<x_{\text {min }}$ carry only a small part of the total momentum. For estimation of the number of gluons in central region of rapidity we use the relation (12):

$$
\begin{gathered}
\left(\frac{d N_{g}}{d y}+\frac{d N_{\text {sea }}}{d y}+\frac{d N_{\text {val }}}{d y}\right)_{y=0} \simeq \\
\simeq \frac{d N_{g}}{d y}+0.44 \frac{d N_{g}}{d y}+\frac{d N_{\text {val }}}{d y} \simeq 1374,
\end{gathered}
$$

where $N_{\text {val }}=3(N-\bar{N}) \simeq 49$ (where $N-\bar{N} \simeq 16.3[5]$ is the number of net nucleons for RHIC in central region of rapidity).

Hence, the estimate for gluons is:

$$
\left(\frac{d N_{g}}{d y}\right)_{y=0} \simeq 920 .
$$


Taking into account Eq. (8), we have the relation for determination of the hot glue initial temperature $T_{g}$ :

$$
n_{g} \tau_{g}=\frac{d N_{g}}{d y \pi R_{A u}^{2}} \simeq 12.5\left(m_{\pi}\right)^{2}=\frac{T^{2}}{2.65 \alpha_{s}^{2}(T)+1.125 \alpha_{s}(T)} .
$$

Here the physical values are in the units of $m_{\pi}=139 \mathrm{MeV}$. The function $\alpha_{s}(T)$ is determined by Eq. (9). We find from Eq. (17):

$$
T_{g} \simeq 345 \mathrm{MeV}
$$

From Eq. (10) the initial gluon density is:

$$
n_{g}\left(T_{g}\right) \simeq 19.42 m_{\pi}^{3} .
$$

Thus the time required to achieve the equilibrium for gluons is

$$
\tau_{g} \simeq \frac{12.5}{n_{g}\left(T_{g}\right)} \simeq \frac{0.64}{m_{\pi}} \simeq 0.91 \mathrm{fm} / \mathrm{c} .
$$

The same time for quarks and gluons found in Ref. [5] is $\tau_{0} \simeq 1.54 / m_{\pi} \simeq 2.18 \mathrm{fm} / \mathrm{c}$. This agrees with estimate in Ref. [6].

In the region $T_{0}<T \leq T_{g}$ the value $\tau_{g}$ we estimate also by Eq. (8) . Such estimates are reasonable, as in the effective quasiparticle model the coupling constant $\alpha_{s}(T)$ decreases with decrease of temperature, and Eqs. (6) - (8) in the lowest order are a good approximation. In fact, in the effective quasiparticle isentropic model we have the initial conditions in plasma: $T_{0} \simeq 219.6 \mathrm{MeV}$ and $\tau_{0} \simeq 1.54 / m_{\pi}[5]$. However, from Eq. (8) at $T=219.6 \mathrm{MeV}$ and $n_{g}=3.7 m_{\pi}^{3}$ (for the case of equilibrium quarks [5] , see Table I) we have $n_{g} \tau_{g}=5.67 m_{\pi}^{2}$, which gives the same value $\tau_{g}=1.53 / m_{\pi}$. Thus Eq. (8) provides matching of values $\tau$ for the two mentioned regions.

The values $\tau$ for different values of $T$ are shown in Table I. In this table we also give the estimates of equilibrium light quark density in the region $T_{0}<T<T_{g}$, where the total equilibrium of gluons and quarks is not achieved (we assume that in the initial state of the hot glue at $T_{g}=345 \mathrm{MeV}$ all the quarks are nonequilibrium ones)

These estimates can be done in the following way:

1. We calculate the number of gluons $N_{g}=n_{g} \tau_{g} \pi R_{A u}^{2}$ in the region of the hot glue $T_{0}<T<T_{g}$. The number of the nonequilibrium quarks $N_{q+s}$ we estimate using Eq. (15) and subtracting the value $N_{g}$. 
2. For these quarks we calculate the nonequilibrium entropy $S_{q+s}$ in the Boltzmann approach (the expected difference for calculations with fermions is about $\sim 5 \%$ ). We calculate also the entropy $S_{g}$ for equilibrium gluons. We assume, that the total entropy $S_{g}+S_{q+s}$ increase smoothly (almost linearly like $N_{q+s}(T)$ ) to the equilibrium entropy $S_{0}\left(T_{0}\right) \simeq 6300$ with decrease of $T \rightarrow T_{0}[5]$. Using this assumption, we estimate the equilibrium addition from quarks to the total entropy density, and equilibrium addition $n_{q}(T)$ to quark density in the region $T_{0}<T<T_{g}$. The equilibrium densities $n_{g}(T)$ and $n_{q}(T)$ in Table $\square$ (at $\left.T_{c} \leq T \leq T_{0}\right)$ we find, using the formulas of effective quasiparticle model [ $[$ ].

The dominating first order radiation intensity distribution for expanding plasma is given in Ref. [4]:

$$
\begin{gathered}
\frac{d I}{d x}=\frac{9 C_{R} E}{\pi^{2}} \int_{z_{0}}^{\infty} d z \rho(z) \int_{|k|_{\min }}^{|k|_{\max }} d^{2} \mathbf{k}, \alpha_{s} \\
\int_{0}^{q_{\max }} d^{2} \mathbf{q} \frac{\alpha_{s}^{2}}{\left[\mathbf{q}^{2}+\mu^{2}(z)\right]^{2}} \frac{\mathbf{k q}}{\mathbf{k}^{2}(\mathbf{k}-\mathbf{q})^{2}} \times \\
\times\left[1-\cos \frac{(\mathbf{k}-\mathbf{q})^{2}\left(z-z_{0}\right)}{2 x(1-x) E}\right],
\end{gathered}
$$

where $E$ is jet energy, and $C_{R}$ is color factor of jet $\left(C_{R}=N_{c}\right.$ for gluons). Here we take into account substitution $x \rightarrow x(1-x)$ for $x \rightarrow 1$ [14]. It is assumed that quark-gluon plasma can be modeled by well separated color-screened Yukawa potentials. The upper kinematic bound of medium induced momentum transfer is $|q|_{\max } \approx \sqrt{3 \mu(\tau) E}$. The transverse momentum $\mathbf{k}_{\perp}^{2}$ is connected with gluon emission distribution from parton jet in the absence of a medium. The kinematic bound on the transverse momentum is $\mathbf{k}_{\max }^{2}=\min \left[4 E^{2} x^{2}, 4 E^{2} x(1-x)\right]$ and $\mathbf{k}_{\min }^{2}=\mu^{2}(\tau)$ for gluons with light cone momentum fraction $x\left(\right.$ where $\mu^{2}(\tau)=4 \pi \alpha_{s} T^{2}(\tau)$ )

The value $z=\tau$ is limited by thickness $\mathrm{L}$ of target, but as a matter of fact, by end of plasma phase and by beginning of mixed phase $\tau_{c}$, i.e. by duration of plasma phase $\tau_{0} \leq \tau \leq \tau_{c}$. The value $\tau_{c}$ does not exceed the radius $R_{A u}$ (or it is close to it). The value $\rho(\tau)$ is the gluon or quark density at time $\tau$ along the jet path (i.e. $\rho=n_{g}$ or $n_{q}$ ). The 
energy loss $\Delta E$ is defined by integration of $d I / d x$ in Eq. (21) over $x$. The value

$$
\begin{gathered}
I_{1}(E, \tau(T))=\int_{0}^{1} d x \int_{|\mathbf{k}|_{\min }}^{|\mathbf{k}|_{\max }} d^{2} \mathbf{k} \alpha_{s}(\tau) \\
\int_{0}^{|\mathbf{q}|_{\max }} \frac{d^{2} \mathbf{q} \alpha_{s}^{2}(\tau)}{\left[\mathbf{q}^{2}+\mu^{2}(\tau)\right]^{2}} \frac{\mathbf{k q}}{\mathbf{k}^{2}(\mathbf{k}-\mathbf{q})^{2}} \times \\
\times\left[1-\cos \frac{(\mathbf{k}-\mathbf{q})^{2} \tau}{2 x(1-x) E}\right]
\end{gathered}
$$

in the finite kinematic bounds is calculated by Monte Carlo method for values of $\tau(T)$ in the region $\tau_{g} \leq \tau \leq \tau_{c}$ (for RHIC energy) and for several values of $E$. In the calculations of complete energy loss $\Delta E$ the scattering of gluons (and quarks) by gluon and quark potentials in plasma medium should be taken into account for every value of $E$. For example, the fraction of gluon scattering on gluon potential in medium is defined by formula:

$$
\frac{n_{g} \sigma_{g g}}{n_{g} \sigma_{g g}+n_{q} \sigma_{g q}}=\frac{1}{1+\frac{n_{q} \sigma_{\sigma q}}{n_{g} \sigma_{g g}}},
$$

where $\sigma_{g g}$ is the cross section for gluon scattered on gluon potential, and $\sigma_{g q}$ is correspondingly cross section for gluon scattering on quark potential. Also the fraction of gluon scattering on quark potential is $\left(1+\frac{n_{g} \sigma_{g g}}{n_{q} \sigma_{q g}}\right)^{-1}$. Taking into account the decrease of the color factor we have $\sigma_{q g} / \sigma_{g g}=4 / 9$ [3] .

The complete energy loss $\Delta E$ of gluon jet is determined by formula:

$$
\begin{aligned}
\Delta E_{g}(E) & =\frac{9 C_{R} E}{\pi^{2} \mu^{2}} \int_{\tau_{g}}^{\tau_{c}} d \tau I_{0}(E, \tau) \frac{n_{g}}{1+\frac{4 n_{q}}{9 n_{g}}}+ \\
& +\frac{4 C_{R} E}{\pi^{2} \mu^{2}} \int_{\tau_{g}}^{\tau_{c}} d \tau I_{0}(E, \tau) \frac{n_{q}}{1+\frac{9 n_{g}}{4 n_{q}}}
\end{aligned}
$$

where the value $I_{0}(E, \tau)$ corresponds to $I_{1}(E, \tau)$ in variable $|k| / \mu$ and $|q| / \mu$. Similarly, the energy loss of quark jet is determined by formula:

$$
\begin{aligned}
\Delta E_{q}(E) & =\frac{4 C_{F} E}{\pi^{2} \mu^{2}} \int_{\tau_{g}}^{\tau_{c}} d \tau I_{0}(E, \tau) \frac{n_{g}}{1+\frac{4 n_{q}}{9 n_{g}}}+ \\
& +\frac{16 C_{F} E}{9 \pi^{2} \mu^{2}} \int_{\tau_{g}}^{\tau_{c}} d \tau I_{0}(E, \tau) \frac{n_{q}}{1+\frac{9 n_{g}}{4 n_{q}}},
\end{aligned}
$$


where $C_{F}$ is the color factor of quark: $C_{F}=\frac{N_{c}^{2}-1}{2 N_{c}}$.

However it should be noted, that for the well-separated color screened Yukawa potentials the condition $\lambda=\frac{1}{\sigma \rho} \gg 1 / \mu$ must be implemented (here $\lambda$ is the mean free path of partons, and $\mu$ is the color screening parton mass). This condition is well realized for parton scattering on gluon potential, where $\mu_{g}^{2} \simeq 4 \pi \alpha_{s} T^{2}$, and we have $\lambda_{g, q} \mu_{g} \gg 1$. But this condition is not realized for partons scattering on the quark Yukawa potential, where $\mu_{q}^{2}=4 \pi \alpha_{s} T^{2} / 6$, and we have here $\lambda_{g, q} \mu_{q} \leq 1$. Therefore the estimation of partons energy loss $\Delta E$ on gluon Yukawa potential is the most real in this model. These energy loss $\Delta E$ of gluon jet we give in Table \for example at $\mathrm{E}=10 \mathrm{GeV}$. But calculating also the energy loss on quark potential by formulas (24- 25), we find the decrease of $\Delta E$ no more than $10 \%$.

The complete energy loss $\Delta E$ is calculated by numeral integration over $\tau$. We have $\Delta E_{g} \simeq 3.15 \mathrm{GeV}$ at $E=10 \mathrm{GeV}$. The complete energy loss of gluon jets $\Delta E_{g}(E)$ is shown in Fig. 1 opposite $E$ (Fig. 2 shows the relative energy loss $\Delta E(E) / E$ ).

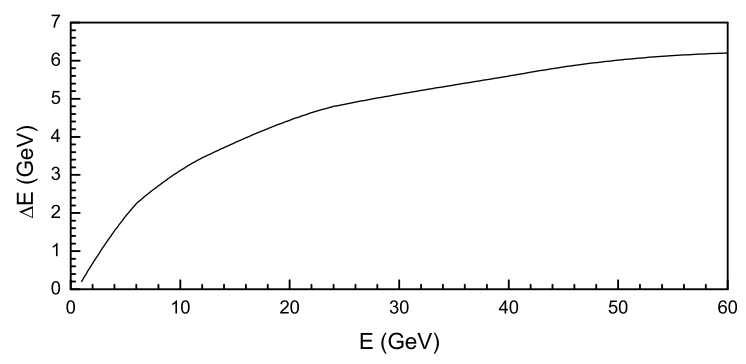

Figure 1: The energy loss of gluon jet in quark-gluon plasma at RHIC energy.

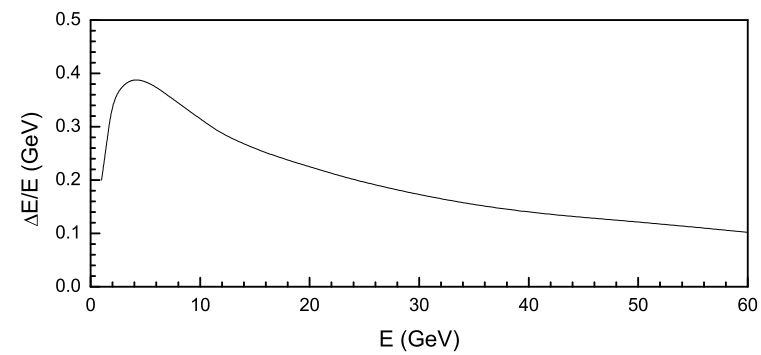

Figure 2: The relative energy loss of gluon jet in quark-gluonf plasma.

Let us consider now the energy loss at SPS energy. For the value $d N / d y$ per unit central 
Table I: The values of various physical quantities for expanding plasma at RHIC energy and energy loss of gluon jet $\Delta E$.

\begin{tabular}{ccccccc}
\hline \hline$T(\mathrm{MeV})$ & $\tau\left(m_{\pi}^{-1}\right)$ & $\alpha_{s}$ & $I_{0}$ & $n_{g}\left(m_{\pi}^{3}\right)$ & $n_{q}\left(m_{\pi}^{3}\right)$ & $\Delta E(\mathrm{GeV})$ \\
\hline 345 & 0.645 & 0.267 & 0.092 & 19.42 & 0 & 2.367 \\
325 & 0.70 & 0.265 & 0.088 & 15.95 & 4.41 & 2.097 \\
300 & 0.79 & 0.262 & 0.085 & 12.22 & 4.71 & 1.855 \\
275 & 0.908 & 0.26 & 0.083 & 9.09 & 4.45 & 1.585 \\
250 & 1.075 & 0.257 & 0.081 & 6.5 & 4.21 & 1.38 \\
225 & 1.34 & 0.252 & 0.079 & 4.4 & 3.50 & 1.13 \\
219.6 & 1.54 & 0.247 & 0.078 & 3.7 & 6.1 & 1.02 \\
210 & 1.74 & 0.241 & 0.077 & 3.12 & 5.134 & 0.964 \\
200 & 2.04 & 0.235 & 0.075 & 2.56 & 4.195 & 0.84 \\
190 & 2.44 & 0.229 & 0.073 & 2.045 & 3.32 & 0.778 \\
185 & 2.72 & 0.222 & 0.067 & 1.795 & 2.9 & 0.663 \\
180 & 3.1 & 0.215 & 0.063 & 1.545 & 2.47 & 0.589 \\
175 & 3.7 & 0.201 & 0.055 & 1.286 & 2.03 & 0.48 \\
172 & 4.26 & 0.184 & 0.043 & 1.116 & 1.73 & 0.37 \\
170 & 4.6 & 0.057 & 0.00067 & 1.23 & 1.61 & 0.0213 \\
\hline \hline
\end{tabular}

rapidity (charged + neutral) we have [5] :

$$
\left(\frac{d N}{d y}\right)_{y=0} \simeq 803 .
$$

The number of partons from structure function is also obtained by Eqs. (13), (14), but now $x_{\text {min }} \simeq 0.09-0.1$. The fraction of sea quarks is $N_{\text {sea }} / N_{g} \simeq 0.58$. Taking into account the number of valent quarks in the central region of rapidity $\simeq 57 \times 3$ we have now:

$$
\left(\frac{d N_{g}}{d y}\right)_{y=0} \simeq 400 .
$$

We use the relation analogous to (17) for derivation of the hot glue initial temperature $T_{g}$ :

$$
\frac{1}{\pi R_{P b}^{2}}\left(\frac{d N_{g}}{d y}\right) \simeq 5.1
$$

It can be shown, however, that there is no solution of equation (17) for $T_{g}$ in that case. Apparently, at such low energy the hot glue phase is practically indistinguishable from 
Table II: The same as Table I but for SPS energy.

\begin{tabular}{ccccccc}
\hline \hline$T(\mathrm{MeV})$ & $\tau\left(m_{\pi}^{-1}\right)$ & $\alpha_{s}$ & $I_{0}$ & $n_{g}\left(m_{\pi}^{3}\right)$ & $n_{q}\left(m_{\pi}^{3}\right)$ & $\Delta E(\mathrm{GeV})$ \\
\hline 175 & 2.4 & 0.201 & 0.061 & 1.28 & 2.2 & 0.212 \\
174 & 2.52 & 0.197 & 0.058 & 1.224 & 2,1 & 0.204 \\
173 & 2.65 & 0.192 & 0.055 & 1.168 & 1.99 & 0.188 \\
172 & 2.8 & 0.184 & 0.049 & 1.11 & 1.88 & 0.168 \\
171 & 2.9 & 0.172 & 0.04 & 1.056 & 1.76 & 0.141 \\
170 & 2.98 & 0.057 & 0.00075 & 1.23 & 1.74 & 0.0091 \\
\hline \hline
\end{tabular}

phase of total equilibrium(or close to it). In Table the energy loss $\Delta E(\tau)$ is shown at SPS energy $(E=4 \mathrm{GeV})$ for the case of total equilibrium. At $E=4 \mathrm{GeV}$ the total energy loss is $\Delta E \simeq 106 \mathrm{MeV}$. Such small energy loss is caused by a short lifetime of the plasma phase at low SPS energy. It should be noted, that the energy losses, which are found here, correspond to the model of phase transition with decrease of effective coupling strength $G(T)$ and mass $m_{g}(T)$ for $T \rightarrow T_{c}$ from above.

The similar problem was considered in the Ref. [5] using the ordinary perturbation theory for powers of running coupling $\alpha_{s}$ in quark-gluon plasma (up to order $O\left(\alpha_{s}\right)$ ). In this approach the coupling $\alpha_{s}$ increases with $T \rightarrow T_{c}$ above. Although this model disagrees with $\mathrm{SU}(3)$ lattice data in the region of phase transition (i.e. close to $T_{c}$ ), we do not find here a noticeable difference for spectra of particles in comparison with the effective quasiparticle model (i.e.the spectra weakly depend on structure of phase transition).

However, let us consider the energy loss at RHIC in this perturbative model. We use the Eq. (8) with running coupling $\alpha_{s}$ [5] (with $\lambda \simeq 180 \mathrm{MeV}$ ) and values $n_{g}$ without quarks and with quarks in the perturbative decomposition. We find the initial temperature of the hot glue to be $T_{g} \simeq 400 \mathrm{MeV}$. The initial temperature of the total equilibrium is $T_{0} \simeq 219 \mathrm{MeV}$, $\tau_{0} \simeq 2.18 \mathrm{fm}$ and $\tau_{c} \simeq 3.73 \mathrm{fm}[5]$. The total energy loss can be found from corresponding Table, which analogous to Table —. For example, we find $\Delta E \simeq 13.5 \mathrm{GeV}$ for $E=16$ $\mathrm{GeV}, \Delta E \simeq 8.5 \mathrm{GeV}$ for $E=10 \mathrm{GeV}$, and $\Delta E \simeq 3.5 \mathrm{GeV}$ for $\mathrm{E}=4 \mathrm{GeV}$. These energy losses as too high and disagree with the data for suppression of hadrons with large $p_{\perp}$ in the central nuclear collisions at RHIC energy. This disagreement is connected with injustice of perturbation theory in region of phase transition. In Ref. [5] the quasiparticle model with 
phenomenological parametrization of coupling constant $G(T)$ was also considered. In this model $G(T)$ increase for $T \rightarrow T_{c}$ from above. This model agrees with the new lattice data and provide also good description of the baryon and meson spectra. However, it can be shown that in this model we have a paradox result for energy loss of gluon jet in plasma: the energy loss exceeds the energy of jet itself (even without taking into account the effect of the hot glue). This contradiction is caused by too large value of coupling $G(T)$, especially close to the phase transition point.

\section{SUPPRESSION OF PIONS WITH LARGE $p_{\perp}$ IN CENTRAL AU+AU COL- LISIONS}

The jet quenching reduced the jet energy before fragmentation, where the jet transverse momentum is shifted by energy loss on to value $\Delta E(E)[15]$. To account for this effect we

should replace the vacuum fragmentation function by effective one $z_{c}^{*} / z_{c} D_{h / c}\left(z_{c}^{*}, \hat{Q}^{2}\right)$, where

$$
z_{c}^{*}=\frac{z_{c}}{1-\frac{\Delta E(E)}{E}} .
$$

The invariant cross section of hadron production in central $\mathrm{A}+\mathrm{A}$ collisions is given by:

$$
\begin{aligned}
& E_{h} \frac{d \sigma_{h}^{A A}}{d^{3} p}=\int_{0}^{b \max } d^{2} b d^{2} r t_{A}(r) t_{A}(|\mathbf{b}-\mathbf{r}|) \\
& \sum_{a b c d} \int d x_{a} d x_{b} d^{2} k_{\perp, a} d^{2} k_{\perp, b} \times \\
\times & g_{A}\left(k_{\perp, a}, Q^{2}, r\right) g_{A}\left(k_{\perp, b}, Q^{2},|\mathbf{b}-\mathbf{r}|\right) \times \\
\times & f_{a / A}\left(x_{a}, Q^{2}, r\right) f_{b / A}\left(x_{b}, Q^{2},|\mathbf{b}-\mathbf{r}|\right) \times \\
\times & \frac{d \sigma}{d \hat{t}} \frac{z_{c}^{*}}{z_{c}} \frac{D_{h / c}\left(z_{c}^{*}, \hat{Q}^{2}\right)}{\pi z_{c}} .
\end{aligned}
$$

Here $t_{A}(r)$ is the nuclear thickness function, $k_{\perp, a}$ and $k_{\perp, b}$ are the initial transverse momenta of partons, $f_{a / A}$ and $f_{b / A}$ are parton structure functions. It is usually assumed that distribution $g_{A}\left(k_{\perp}\right)$ has a Gaussian form.

It should be noted, that intrinsic $k_{\perp}$ and the transverse momentum broadening (Cronin effect) are important for final hadron spectra for SPS energies. However, with the increase of energy the spectra become flatter and small initial $k_{\perp}$ correspond to small variation of spectra. At RHIC energy one can neglect the effects of initial $k_{\perp}$ with a good precision 
16]. In this work we do not take into account the intrinsic transverse momentum at RHIC energy.

For parton distribution of nucleon in nucleus we take into account the parton shadowing factor $S_{a, b / A}(x, r)$ for spherical nucleus and $S_{a, b / A}(x)$ for flat disk, for which we take the parametrization used in HIJING model [17]. In this model the factor $S_{a / A}(x, r)$ is splitted into two parts: $S(x, r)=S_{0}(x)-\alpha_{A}(r) S_{1}(x)$, where $\alpha_{A}(r)=0.1\left(A^{1 / 3}-1\right) 4 / 3 \sqrt{1-r^{2} / R_{A}^{2}}$. For flat disk we have $\alpha_{A}(r) \rightarrow \bar{\alpha}_{A}=0.1\left(A^{1 / 3}-1\right)$.

The upper limit for the impact parameter is $b_{\max } \simeq 0.632 R_{A u}$ for $10 \%$ central $\mathrm{Au}+\mathrm{Au}$ collisions. Neglecting initial $k_{\perp}$ in Eq. (30) it is convenient to introduce the new variable $x_{a} \equiv x_{1}=x_{\perp}^{\pi} \xi / 2 z, x_{b} \equiv x_{2}=x_{\perp}^{\pi} \xi / 2 z(\xi-1)$, where $x_{\perp}^{\pi}=2 p_{\perp}^{\pi} / \sqrt{s}, z=x_{\perp}^{\pi} / x_{\perp}, x_{\perp}=2 E / \sqrt{s}$, i.e. $z=p_{\perp}^{\pi} / E$. After integration over $r$ and $b$ in Eq. (30) we obtain cross section for hadron production in gluon jet in the central $\mathrm{Au}+\mathrm{Au}$ collision:

$$
\begin{aligned}
E_{h} \frac{d \sigma_{h}^{A A}}{d^{3} p} & =\frac{9 K}{\left(p_{\perp}^{\pi}\right)^{4}} \int_{x_{\perp}^{\pi}}^{z_{\max }} d z \alpha_{s}^{2}(Q(z)) z^{2} D_{h / g}\left(z_{c}^{*}, \hat{Q}^{2}\right) \frac{1}{\left(1-\frac{\Delta E}{E}(z)\right.} \times \\
& \times \int_{\frac{2 z}{2 z-x_{\perp}^{\pi}}}^{\frac{2 z}{x_{\perp}^{\pi}}} d \xi f^{g g}(\xi) \Phi\left(x_{1}, x_{2}\right) x_{1} G_{g}\left(x_{1}\right) x_{2} G_{g}\left(x_{2}\right),
\end{aligned}
$$

where $Q=p_{\perp}^{\pi} / 2 z, \hat{Q}=p_{\perp}^{\pi} / 2 z^{*}$, and $G_{g}\left(x_{1}\right), G_{g}\left(x_{2}\right)$ are the gluon structure functions. $D_{h / g}$ is fragmentation function [18] and $K$ is K-factor. Function $\Phi\left(x_{1}, x_{2}\right)$ describes the contribution of shadowing. For spherical nuclei (for $10 \%$ central collisions) we obtain: $\Phi\left(x_{1}, x_{2}\right)=$ $226.58 S_{0}\left(x_{1}\right) S_{0}\left(x_{2}\right)-117.27 S_{1}\left(x_{1}\right) S_{0}\left(x_{2}\right)-97.69 S_{0}\left(x_{1}\right) S_{1}\left(x_{2}\right)+54.87 S_{1}\left(x_{1}\right) S_{1}\left(x_{2}\right)$. Functions $S_{0}$ and $S_{1}$ can be found in Ref. [17]. The value $f_{g g}(\xi)$ is the fraction of gluon-gluon elementary cross-section in variable $\xi$ :

$$
f_{g g}(\xi)=\frac{3(\xi-1)}{\xi^{4}}-\frac{(\xi-1)^{2}}{\xi^{6}}+\frac{1}{(\xi-1) \xi^{3}}+\frac{(\xi-1)^{2}}{\xi^{3}} .
$$

We also have:

$$
\begin{gathered}
f_{q g}(\xi)=\frac{1+\xi^{2}}{\xi^{4}(\xi-1)}+\frac{4(\xi-1)}{9 \xi^{5}}+\frac{4(\xi-1)}{9 \xi^{3}} \\
f_{q q}(\xi)=\frac{4\left(1+\xi^{2}\right)}{9 \xi^{4}(\xi-1)}
\end{gathered}
$$


The value $z_{\max }$ can be found from Eq. (29) $): z_{c}^{*}=\frac{p_{\perp}^{\pi}}{E\left(1-\frac{\Delta E}{E}\right)}=\frac{p_{\perp}^{\pi}}{E-\Delta E}=1$ (taking into account that $\left.z=p_{\perp}^{\pi} / E\right)$. The value of $E$ which corresponds to gluon jet with some $p_{\perp}^{\pi}$ can be found from Fig. 1(in large scale). For example, for values $p_{\perp}^{\pi}=3,4,6 \mathrm{GeV} /$ c we can estimate $z_{\max }=0.61,0.64,0.67$ (e.g. at $p_{\perp}=4$ we have $E-\Delta E(E) \simeq 6.25-2.25=4$, and $\left.z_{\text {max }} \simeq 4 / 6.25=0.64\right)$.

We obtain the approximate numeral formulas for $\frac{\Delta E}{E}(z)$ in the region $z_{\min }<z<z_{\max }$ for several values of $p_{\perp}^{\pi}$ (which are $(3-5) \%$ precise). For example, for $p_{\perp}^{\pi}=4 \mathrm{GeV}$ we have (here $\left.z_{\min }=2 P_{\perp} / \sqrt{s} \simeq 0.0615\right)$ :

$$
\frac{\Delta E}{E}(z) \simeq 0.46 z+0.061+0.95(z-0.0615)(0.64-z) .
$$

The relations of this kind we use in formula (31) for gluon jets.

But it should be noted, that for the quark scattering on gluon and quark potentials the color factor decrease $16 / 81=0.198$ times and the energy loss $\Delta E$ should decrease correspondingly. From relation $E-0.198 \Delta E_{g}=p_{\perp}^{\pi}$ we can find for $p_{\perp}^{\pi}=3,4,6 \mathrm{GeV}$ the corresponding values $z_{\max } \simeq 0.91,0.92,0.924$. Here we also find the approximate analytical formulas for $\frac{\Delta E}{E}(z)$, which we use in Eq. (31) for quark jets. For example at $p_{\perp}^{\pi}=4 \mathrm{GeV}$ we have:

$$
\frac{\Delta E}{E}(z) \simeq 0.068 z+0.014+0.11(z-0.0615)(0.92-z)
$$

In this work we calculate the effective suppression factor for the $\pi^{0}$ spectra at RHIC energy, or the ratio:

$$
R_{A A}\left(p_{\perp}\right)=\frac{\frac{d N_{A A}}{d y d^{2} p_{\perp}}}{\sigma_{i n}^{p p} \frac{d N_{p p}}{d y d^{2} p_{\perp}} \bar{T}}
$$

where $\bar{T}=\int_{0}^{b_{\max }} T(b) d^{2} b / \int_{0}^{b_{\max }} d^{2} b$. This is ratio between the spectrum in central AA and pp collisions, which is normalized to the effective total number of binary NN collision in central AA collisions. If we do not take into account the nuclear effects (jet quenching and shadowing), this ratio should be unity at large $p_{\perp}$.

In Eq. (31) for invariant cross section we take into account the following parton collisions:

1. The gluon-gluon collision, the gluon scattering by gluon potential and gluon fragmentation into $\pi^{0}$. This process is described by Eq. (31) with effective fragmentation function $D_{\pi^{0} / g}\left(z_{c}^{*}, \hat{Q}^{2}\right)$ [18] and with function $\frac{\Delta E}{E}(z)$ of the kind (35) for each value of $p_{\perp}^{\pi}$. 
2. The gluon-quark collision (with $\mathrm{u}$ and d quarks), the gluon scattering by gluon and quark potentials and fragmentation of gluon. In Eq. (311) we use $f_{q g}(\xi)$ from (33) with corresponding factor, the product $G_{g} G_{q}$ from [12] and $\Delta E / E$ of the kind (35).

3. The $q g$ collision, the quark scattering by gluon and quark potentials and fragmentation of quark into $\pi^{0}$. In Eq. (31) we have now $D_{\pi^{0} / q}[18]$ with $\frac{\Delta E}{E}(z)$ of the kind (36) for each value of $p_{\perp}^{\pi}$ due to the decrease of the color factor.

4. The quark-quark collision $f_{q q}(\xi)$ (34), the quark scattering by gluon and quark potentials and fragmentation of quark. We have here $\Delta E / E$ also of type (36) and product $G_{q} G_{q}$.

5. The collision of gluon with sea quarks, the gluon scattering by gluon and quark potentials and gluon fragmentation. Here we also use the effective fragmentation function $D_{\pi^{0} / g}$ with $\Delta E / E$ of the kind (35) and corresponding structure function from Ref. [12].

In order to find the effective suppression factor $R_{A A}\left(p_{\perp}\right)$ of the $\pi^{0}$ spectra, the cross section (31) should be divided by the same cross section for the same value of $p_{\perp}^{\pi^{0}}$, but without nuclear effects $\left(\Delta E=0, z_{\max }=1\right)$, and without shadowing $\left(S_{0}\left(x_{1}\right)=S_{0}\left(x_{2}\right)=1\right.$, $S_{1}\left(x_{1}\right)=S_{1}\left(x_{2}\right)=0$ for spherical nuclei).

We have calculated such ratio for hard collisions $\left(3 \mathrm{GeV} / \mathrm{c} \leq p_{\perp}^{\pi^{0}} \leq 6 \mathrm{GeV} / \mathrm{c}\right)$. For example, this ratio for $p_{\perp}^{\pi^{0}}=4 \mathrm{GeV} / \mathrm{c}$ (the contributions of collisions listed above in items $1-5$ are given in numerator and denominator) is:

$$
\begin{aligned}
& R_{A A}\left(p_{\perp}^{\pi^{0}}=4 \mathrm{GeV} / \mathrm{c}\right)= \\
= & \frac{0.37+0.08+1.09+0.27+0.015}{4.0+0.53+1.8+0.40+0.161} \simeq 0.27 .
\end{aligned}
$$

The values of $R_{A A}$ corresponding to $p_{\perp}^{\pi^{0}}=3,4,5,6 \mathrm{GeV}$ are $R_{A A}=0.23,0.27,0.32,0.38$. We show in Fig. 3 the ratio $R_{A u, A u}\left(p_{\perp}^{\pi^{0}}\right)$ and the experimental data at RHIC energy. The theoretical values of $R_{A u, A u}$ are somewhat smaller, than the experimental values, though they apparently are within the limits of experimental uncertainly.

Theoretical values of $R_{A A}$ in Fig. [ correspond to sufficiently large values of $p_{\perp}$. In the intermediate region $1 \leq p_{\perp} \leq 3 \mathrm{GeV}$ there are uncertainties associated with the interplay of contributions from hard and soft processes. In the soft region $p_{\perp}<1 \mathrm{GeV} / \mathrm{c}$ it is possible 


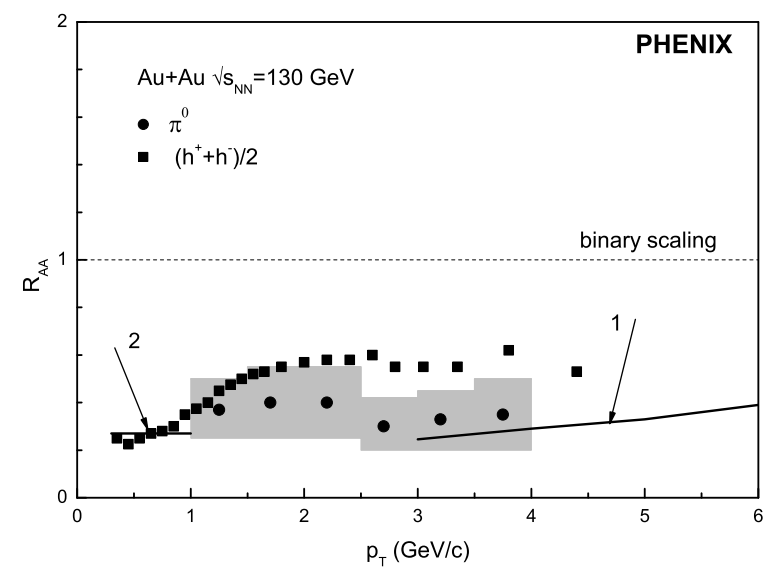

Figure 3: The suppression factor $R_{A A}\left(p_{\perp}\right)$ for charged hadrons and neutral pions. The gray band for $\pi^{0}$ is defined by the sums of squares of the systematic errors of the measurement and uncertainty in the $N+N$ reference and in $\left\langle N_{\text {binary }}\right\rangle$. The errors for $\left(h^{+}+h^{-}\right) / 2$ are not shown. The line 1 is the calculated factor $R_{A A}\left(p_{\perp}^{\pi^{0}}\right)$ for hard processes, the line 2 is factor for soft processes calculated in assumption that the low $p_{\perp}$ spectra scale like $A^{1.1}$.

make the estimate of $R_{A A}$ by Eq. (37) if the scaling $A^{1.1}$ is used: $\frac{d N_{A A}}{d y} \simeq A^{1.1} \frac{d N_{p p}}{d y}$. This gives the value $R_{A A} \simeq 0.25-0.29$, which is close to experimental data available for charged particles.

\section{CONCLUSION}

In this paper we investigate the energy loss of quark and gluon jets in the quark-gluon plasma and suppression of the $\pi^{0}$ spectra at RHIC energy. We use the initial conditions in plasma, which were found earlier in the effective quasiparticle model for SPS and RHIC energies [5]. We take into account also the possibility of production of the hot glue at the first stage with more high temperature and density. It can be shown, that plasma is sufficiently thin: $\bar{n}=\int_{\tau_{g}}^{\tau_{c}} d \tau n_{g}(\tau) \sigma_{g g}(\tau) \simeq 1.28$, where $\bar{n}$ is an average number of jet scatterings. The energy loss in expanding plasma is calculated in the dominant first order [3, 4] taking into account the finite kinematic limits. The energy losses $\Delta E$ of gluon and quark jets are calculated in a wide range of parton energies $E$. These energy losses are used for calculation of suppression of $\pi^{0}$ spectra with moderately high $p_{\perp}\left(3<p_{\perp}<6 \mathrm{GeV} / \mathrm{c}\right)$, which is done 
by modification of gluon and quark fragmentation functions. We also take into account the parton shadowing factors in nucleus [17]. We obtain a considerable suppression of $\pi^{0}$ spectra, which is caused by the effects mentioned above. This suppression agrees with the data reported by PHENIX in the region $p_{\perp}^{\pi_{0}}=3-4 \mathrm{GeV} / \mathrm{c}$. The estimates of $R_{A A}$ (37) in the soft region $\left(p_{\perp}<1 \mathrm{GeV} / \mathrm{c}\right)$ also agrees with experimental data if the scaling $A^{1.1}$ is taken into account.

The intrinsic transverse momentum $k_{\perp}$ at RHIC energy is neglected in this work. It is important at SPS energy (Cronin effect), but at RHIC energy one can neglect the effects of initial $k_{\perp}$ with a good precision [16]. In principle a small increase of $R_{A A}$ is possible at moderate $p_{\perp}$ because of transverse momentum broadening. We are planning to take into account the intrinsic $k_{\perp}$ in the next work.

The most important conclusion of this work is the possibility to investigate the structure of the plasma phase transition into hadrons with the help of hard processes. The correct quantitative description of $\pi^{0}$ suppression is probably possible only in a model of phase transition which includes the decrease of thermal gluon mass $m_{g}(T)$ and effective coupling $G(T)$ near the phase transition $\left(T \simeq T_{c}\right)$ point. However, the quasiparticle model with increase of these values at $T \rightarrow T_{c}$ leads to a too high energy losses (which can even exceed the energy of the jet itself). The main reason of this problem is too large value of the coupling $G(T)$ near $T_{c}$. We show also that energy losses at SPS energy are very small as a consequence of a too low initial temperature of the plasma phase at this energy.

It is interesting to investigate also the suppression of charged meson and baryon spectra, which we are planning to do in the forthcoming paper.

\section{Acknowledgments}

The author thank Profs. S. T. Beljaev, V. I. Manko and especially S. L. Fokin for fruitful discussions. The author is grateful to Prof. B. V. Danilin, Drs. A. Lomonosov, and L. V. Grigorenko for careful reading of the manuscript. The work was supported by the grant NS-1885.2003.2 of the Russian Ministry of Industry and Science.

[1] X.N. Wang, M. Gyulassy, Phys. Rev. Lett. 68, 1480 (1992). 
[2] K. Adcox et.al., Phys. Rev. Lett. 88, v.2 (2002).

[3] M.Gyulassy, P. Levai, I. Vitev, Nucl. Phys. B594, 371 (2001).

[4] M. Gyulassy, I. Vitev,and X.N. Wang, Phys. Rev. Lett. 86, 2537 (2001)

P. Levai et.al. Nucl. Phys. A698,631 (2002).

[5] Yu.A. Tarasov, hep-ph/0307300, (2003).

[6] E. Shuryak, Phys. Rev. Lett. 68, 3270 (1992)

E.Shuryak, L. Xiong, Phys. Rev. Lett. 70, 2241 (1993).

[7] A. Peshier et.al. Phys. Rev. D54, 2339 (1996).

[8] A. Peshier, B. Kampfer, G. Soff, Phys. Rev. C61, 045203 (2000), hep-ph/0106090

[9] R.A. Schneider, W. Weise, Phys. Rev. C64, 055201 (2001).

[10] J. Combridge, J. Kripfganz, and J. Ranft, Phys. Lett. 70, 234 (1977).

[11] J.D. Bjorken, Phys. Rev. D27, 140 (1983).

[12] M. Gluck, E. Reya, A. Vogt, Zeit. Phys. C67, 433 (1995).

[13] R.P.Feynman Photon-Hadron Interaction, Massachusetts, W.A.Benjamin, Inc.1972

[14] R. Baier et.al. Nucl. Phys. B531, 403 (1998)

B.G. Zakharov, hep-ph/0012360, (2000).

[15] X.N. Wang and Z. Huang, Phys. Rev. C55, 3047 (1997).

[16] X.N. Wang, Phys. Rev. C61, 064910 (2000).

[17] X.N. Wang and M. Gyulassy, Phys. Rev. D44, 350 (1991).

[18] J. Binnewies, B.A. Kniehl, G. Kramer, Phys. Rev. D52, 4947 (1995). 